\title{
Producción de briquetas con residuos de cáscara de piñón manso (Jatropha curcas) y bagazo de caña de azúcar
}

\author{
Production of briquettes with Jatropha curcas shell \\ and sugar cane bagasse
}

\author{
Walter Danilo Maradiaga Rodriguez a*, Adão Wagner Evangelista a, Carlos R Sette Jr a, \\ José Alves Júnior a, Macksuel Fernandes da Silva ${ }^{\text {a }}$ \\ *Autor de correspondencia: ${ }^{a}$ Universidad Federal de Goiás, Escuela de Agronomía, Goiânia, Brasil, \\ tel.: +55-62-35211530, maradiagarodriguez@yahoo.com
}

\begin{abstract}
SUMMARY
One of the alternatives to benefit the agroforestry residues is by densification processes. Thus, the goal of this analysis was to evaluate the raw material and briquettes quality produced from the remains of the sugarcane bagasse and Jatropha curcas shell. The biomass was dried and set at $12 \%$ of moisture content and, afterwards, had the treatments prepared. T1 - $100 \%$ sugar cane bagasse; T2 - $100 \%$ Jatropha curcas shell; T3 - $75 \%$ sugar cane bagasse + $25 \%$ Jatropha curcas shell; T4 - $75 \%$ Jatropha curcas shell $+25 \%$ sugar cane bagasse; and T5 - 50\% sugar cane bagasse $+50 \%$ Jatropha curcas shell. The briquettes were produced with the assistance of a hydraulic briquette machine at temperature of $80 \pm 2{ }^{\circ} \mathrm{C}$ and pressure of $140 \mathrm{kgf} \mathrm{cm}^{-2}$, kept for a period of five minutes. The main energy and physic mechanical aspects of the briquettes were bulk density, energy density, volumetric expansion, dynamic resistance and tensile strength by diametric compression. It was confirmed that the briquettes produced from $100 \%$ Jatropha curcas shell showed superior energy density, they were also more resistant to tensile strength by diametric compression and to the dynamic resistance test, they even presented higher volumetric expansion compared to the ones produced from $50 \%$ sugarcane bagasse $+50 \%$ Jatropha curcas shell.
\end{abstract}

Key words: densified, bioenergy, biomass.

\section{RESUMEN}

La densificación es una alternativa para aprovechar los residuos agroforestales. Así el objetivo del presente estudio fue caracterizar la calidad de la materia prima y briquetas producidas a partir de residuos de bagazo de caña de azúcar y cáscara de piñón manso (Jatropha curcas) en diferentes proporciones. La biomasa fue secada y ajustada al $12 \%$ de humedad y en seguida preparados los tratamientos: T1- $100 \%$ bagazo de caña de azúcar, T2- $100 \%$ cáscara de piñón manso, T3- $75 \%$ de bagazo de caña de azúcar $+25 \%$ cáscara de piñón manso, T4- $75 \%$ cáscara de piñón manso $+25 \%$ bagazo de caña de azúcar y T5- $50 \%$ bagazo de caña de azúcar $+50 \%$ de cáscara de piñón manso. Las briquetas fueron producidas con la ayuda de una briquetadera hidráulica a temperatura de $80 \pm 2{ }^{\circ} \mathrm{C}$ y presión de $140 \mathrm{kgf} \mathrm{cm}^{-2}$, durante cinco minutos. Se analizó densidad aparente, densidad energética, expansión volumétrica, resistencia dinámica y resistencia a la tracción por comprensión diametral. Briquetas confeccionadas con $100 \%$ de cáscara de piñón manso presentaron mayor densidad energética, fueron más resistentes a la prueba de resistencia dinámica y a la tracción por compresión diametral, aunque presentaron mayor expansión volumétrica en relación a las briquetas con $50 \%$ de bagazo de caña de azúcar $+50 \%$ de cáscara de piñón manso.

Palabras clave: densificado, bioenergía, biomasa.

\section{INTRODUCCIÓN}

El aumento de la población mundial, la necesidad de reducir las emisiones de gases de efecto invernadero y la demanda por fuentes de energía limpia, barata y renovable ha llevado a evaluar el potencial energético de varios subproductos agroforestales (Dias et al. 2012). La generación de residuos provenientes de biomasa agroforestal en Brasil es grande, y puede causar problemas ambientales, como inundaciones, contaminar cursos de agua, además de ocupar espacio en las industrias (Protásio et al. 2011).

La caña de azúcar (Saccharum officinarum L.) es uno de los principales cultivos del sistema económico de Brasil y en media durante el proceso de industrialización genera $250 \mathrm{~kg}$ de bagazo por tonelada de caña procesada, residuo que es destinado en su mayoría para la producción de energía térmica en ingenios y destilerías (Cordeiro et al. 2008). Por su parte el piñón manso, es una de las 200 especies 
oleaginosas con potencial para la producción de biodisel en Brasil (Beltrão 2006). Uno de los residuos del piñón manso, que podría utilizarse como fuente energética, es la cáscara de piñón manso. En este sentido, es necesario evaluar el material para cuantificar características físico-químicas y la asociación con otros materiales energéticos, como el bagazo de caña de azúcar, a fin de mejorar su uso, que hasta hoy ha sido poco estudiado (Tomeleri et al. 2017).

Con el actual modelo económico de desarrollo y el avance de las tecnologías de producción, se estima que la producción de caña de azúcar y de piñón manso aumentará y traerá como consecuencia el incremento de residuos de estos cultivos (Oliveira et al. 2012). Con excepción del bagazo de caña de azúcar, el resto de biomasa proveniente de actividades agrícolas, todavía es sub-utilizada para generar energía. En la mayoría de veces es depositada en el campo para descomposición natural, sin aprovechar el potencial energético de algunos materiales, que se convierte en pasivo ambiental (Cortez et al. 1992).

Una alternativa que se presenta para la utilización de residuos agroforestales y reducir el espacio ocupado, es la producción de briquetas. La producción de briquetas, consiste en aplicar presión a una masa de partículas, con o sin tratamiento térmico posterior (Júnior et al. 2014). La generación de energía a partir de briquetas, es influenciada directamente por la calidad de la biomasa, siendo necesario evaluar su calidad (Vale et al. 2011). En este sentido, caracterizar la cáscara de piñón manso y el bagazo de caña de azúcar para uso energético, es un paso importante para determinar, si es posible utilizarla como fuente de energía a través de briquetas.

Las briquetas son constituidas de materiales sólidos generalmente en forma de pequeñas partículas de aserrín, virutas o polvo de madera, prensadas en equipamientos agrícolas o prensas mecánicas con el instinto de obtener un material compacto, generalmente en forma de pequeños cilindros o discos (Júnior et al. 2014).

El uso de briquetas va desde el uso en hornos industriales para elaborar alimentos, como panaderías, pizzerías, churrasquerías, hasta en residencias e industrias de cerámica, donde el tipo y características exigidas para cada uso son variadas (Soares et al. 2015).

Se sabe que no toda la biomasa agroforestal puede ser utilizada para generar energía, debido a que algunos materiales presentan baja densidad de partículas, bajo poder calorífico o alto porcentaje de humedad, ocasionando altos costos durante el transporte y almacenamiento lo que termina inviabilizando su uso (Soares et al. 2015). Así el estudio parte de la hipótesis que mezclar biomasa de caña de azúcar y cáscara de piñón manso (Jatropha curcas L.) en diferentes proporciones, genera briquetas con atributos de calidad tanto para el transporte, almacenamiento y producción de energía. Así el estudio tiene como objetivo caracterizar la calidad de la materia prima y briquetas producidas a partir de residuos de bagazo de caña de azúcar y cáscara de piñón manso.

\section{MÉTODOS}

Para la confección de las briquetas fueron utilizados residuos de cáscara de piñón manso y bagazo de caña de azúcar. El bagazo fue colectado de residuos provenientes de caña de azúcar producida sobre sistema de producción orgánica. La cáscara de frutos de piñón manso, fue colectada de un plantío convencional con cinco años de edad. Para extraer la cáscara de piñón manso, fue realizado el despulpe manual de los frutos. Ambas plantaciones estaban localizadas en la región central del Estado de Goiás, Brasil (16 36' S e $\left.49^{\circ} 17^{\prime} \mathrm{W}\right)$. Según la clasificación climática de Koppen, el clima de la región es Aw (caliente y húmedo), con temperatura media anual, humidad relativa (UR\%) y precipitación de $22,5^{\circ} \mathrm{C}, 71 \%$ y $1.460 \mathrm{~mm}$, respectivamente.

La biomasa colectada fue triturada, utilizando un molino de cuchillas del tipo Willey y posteriormente sometida a separación mecánica mediante un agitador orbital durante 10 minutos. En seguida, la biomasa fue tamizada en una malla de 60 mesh.

El diseño estadístico utilizado fue completamente al azar, con cinco repeticiones. Los tratamientos correspondieron a diferentes proporciones de cáscara de piñón manso y bagazo de caña de azúcar mezclados de la siguiente manera: T1- $100 \%$ bagazo de caña de azúcar, T2- $100 \%$ cáscara de piñón manso, T3- $75 \%$ de bagazo de caña de azúcar + $25 \%$ cáscara de piñón manso, T4- $75 \%$ cáscara de piñón manso $+25 \%$ bagazo de caña de azúcar y T5- $50 \%$ bagazo de caña de azúcar $+50 \%$ de cáscara de piñón manso.

El material molido fue colocado en estufa de circulación forzada a $105^{\circ} \mathrm{C}\left( \pm 2{ }^{\circ} \mathrm{C}\right)$ hasta alcanzar la masa seca constante. En seguida, el material seco fue almacenado en sacos de polietileno a temperatura ambiente $\left(25^{\circ} \mathrm{C} \pm 2{ }^{\circ} \mathrm{C}\right)$ hasta su utilización. La determinación de densidad a granel, se realizó con el material absolutamente seco y fueron constituidas muestras de $500 \mathrm{~cm}^{3}$.

La masa presente en el volumen de cada muestra, fue determinada a través de una balanza analítica con precisión de $0,001 \mathrm{~g}$. La densidad a granel fue obtenida dividiendo la masa seca por el volumen de la muestra. El porcentaje de material volátil, ceniza y carbono fijo, fue evaluado de acuerdo con el cálculo descrito por la norma ASTM D1102-84 (2007). Para la cuantificación del poder calorífico superior (PCS), se utilizó una bomba calorimétrica con base en la norma ASTM D2015-00 (2000).

El material molido fue ajustado al $12 \%$ de humedad, con auxilio de un dosificador de agua y una balanza de precisión. El ajuste de humedad al $12 \%$ realizado, es el recomendado para la fabricación de briquetas (Lucena et al. 2008). Realizado el ajuste de humedad, el material fue almacenado en sacos plásticos, durante 24 horas antes de la confección de las briquetas. Para cada briqueta se utilizó $40 \mathrm{~g}$ de biomasa molida, obteniéndose al final briquetas de aproximadamente $4 \mathrm{~cm}$ de altura y $3 \mathrm{~cm}$ de diámetro. Fueron producidas 10 cuerpos de prueba por tratamiento, totalizando así 50 briquetas para evaluación. 
La compactación de la biomasa fue realizada en una briquetadera hidráulica a temperatura de $80 \pm 2{ }^{\circ} \mathrm{C}$ y presión de $140 \mathrm{kgf} \mathrm{cm}^{-2}$, durante un periodo de cinco minutos. Las briquetas formadas, fueron enfriadas mediante ventilación forzada durante diez minutos. Posteriormente se determinó la densidad aparente $\left(\mathrm{kg} \mathrm{m}^{-3}\right)$, mediante el método estereométrico.

Para determinar el volumen de cada briqueta producida, se realizó medición de altura y diámetro en la parte media de la briqueta, utilizándose para ello un paquímetro digital con precisión de $10^{-3}$ milímetros.

La masa de cada briqueta fue determinada por medio de una balanza analítica con precisión de $10^{-3} \mathrm{~g}$. En seguida se almacenaron en sacos de plásticos para mantener humedad constante. Setenta y dos horas después, tiempo necesario para la estabilización dimensional de las briquetas (Yamaji et al. 2013) fue determinada la expansión volumétrica por medio de la ecuación 1.

$$
\operatorname{Exp}=\left(\mathrm{Vol}_{2}-\mathrm{Vol}_{1}\right) / \mathrm{Vol}_{1} \times 100
$$

Donde,

Exp = expansión volumétrica de las briquetas (\%).

$\mathrm{Vol}_{1}=$ volumen de la briqueta inmediatamente después de la compactación $\left(\mathrm{cm}^{3}\right)$

$\mathrm{Vol}_{2}=$ volumen de la briqueta transcurrido 72 horas de compactación $\left(\mathrm{cm}^{3}\right)$.

La densidad energética fue calculada multiplicando la densidad aparente de la briqueta por el poder calorífico superior. Mientras la densidad energética de la biomasa, fue calculada multiplicando el poder calorífico superior por la densidad a granel. En el caso de la resistencia a la tracción por comprensión diametral, fue evaluada según la norma NBR 7222 (ANBT 1994).

La prueba de resistencia a la tracción por comprensión diametral, fue realizada mediante el uso de cinco briquetas por tratamiento, para lo cual se utilizó una maquina universal de ensayos EMIC - DL30000, con célula de carga y $500 \mathrm{kgf} \mathrm{cm}^{-2}$, a una velocidad constante de $0,3 \mathrm{~min}^{-1}$. Para la prueba de resistencia se aplicó carga en sentido transversal, sobre el cuerpo de prueba y fue realizada a partir de una adaptación de la norma ABNT - NBR 7222 (1994), para muestras cilíndricas de concreto.

La prueba de resistencia dinámica o friabilidad (\%), fue determinada por pérdida de masa de la briqueta. Por cada tratamiento fueron pesados cincos cuerpos de prueba; determinada la masa inicial en gramos, las briquetas fueron llevados a un tamiz vibratorio durante diez minutos a 80 rotaciones por minuto. En seguida, las briquetas fueron pesadas y determinado el porcentaje de durabilidad mediante la ecuación 2.

$$
\text { Durabilidad }=100-\left[\frac{m_{i}-m_{f}}{m_{i}} \times 100 \%\right]
$$

Donde, $\mathrm{m}_{\mathrm{i}}=$ Masa inicial en gramos.

$\mathrm{m}_{\mathrm{f}}=$ Masa final en gramos.

Los datos fueron sometidos a las presuposiciones del análisis de varianza (ANDEVA), posteriormente fueron sometidos a análisis través del programa estadístico JMP. Cuando fue encontrada diferencia estadística significativa entre los tratamientos, se realizó la prueba de Tukey ajustada al $95 \%$ de probabilidad.

\section{RESULTADOS}

Características fisicas y energéticas de la biomasa. Los resultados del análisis de varianza, indicaron diferencias significativas $(P<0,05)$ entre las características físicas y químicas de residuos de cáscara de piñón manso y bagazo de caña de azúcar. La densidad a granel de la biomasa varió entre 170 y $320 \mathrm{~kg} \mathrm{~m}^{-3}$ a pesar de que todos los tratamientos fueron confeccionados con el mismo tamaño de partícula (60 mesh). La cáscara de piñón manso (T2) y el bagazo de caña de azúcar (T1), presentaron mayor y menor densidad a granel respectivamente. La cáscara de piñón manso presentó densidad de $320 \mathrm{~kg} \mathrm{~m}^{-3}$, siendo aproximadamente $88 \%$ mayor que la densidad del bagazo de caña de azúcar $\left(170 \mathrm{~kg} \mathrm{~m}^{-3}\right)$.

El poder calorífico de la biomasa del bagazo de caña de azúcar (figura 1A) presentó 17,14 $\mathrm{MJ} \mathrm{kg}^{-1}$ y la cáscara de piñón manso 14,69 $\mathrm{MJ} \mathrm{kg}^{-1}$. La densidad energética de la biomasa compactada con $100 \%$ de cáscara de piñón manso (figura 1B), incrementó $63 \%$ en relación al tratamiento con $100 \%$ bagazo de caña de azúcar (T1). Los tratamientos T4, T5 y T3, fueron $52 \%, 34 \%$ y $28 \%$ mayores que T1. A medida que aumentó el contenido de bagazo, la densidad energética disminuyó, aunque presentó mayor potencial calorífico en relación al resto de tratamientos.

En relación al contenido de cenizas (figura 2A) se verificó que T3 presentó menor contenido. A medida que disminuyó el contenido de bagazo, aumentó el contenido de ceniza, pasando de $4,4 \%$ (T3) a 14,03\% (T2). El mayor contenido de materiales volátiles ( $89 \%)$ se constató en el tratamiento 1 (figura 2B), siendo $14 \%$ mayor que T2. Los tratamientos T3, T4 y T5, presentaron contenidos inferiores a T1 y superior a T2. En relación con el carbón fijo, T2 presentó mayor porcentaje (7,5\%) (figura $2 \mathrm{C}$ ) en relación a T1 y además fue superior a los tratamientos T3, T4 y T5, respectivamente.

Evaluación energética y físico-mecánica de las briquetas. Los resultados del análisis de varianza indicaron diferencias significativas $(P<0,01)$ entre briquetas producidas con diferentes porcentajes de biomasa de cáscara de piñón manso y bagazo de caña de azúcar (cuadro 1).

La densidad aparente de la briqueta aumentó a medida se incrementó la proporción de cáscara de piñón manso y decreció a medida se aumentó la cantidad de bagazo de caña de azúcar. Para la densidad energética no existió diferen- 
cia estadística entre los tratamientos, aunque matemáticamente se observó que briquetas confeccionadas con $100 \%$ de cáscara de piñón manso, aumentaron la densidad en $17 \%$, en relación a briquetas producidas con $50 \%$ de bagazo de caña de azúcar $+50 \%$ de cáscara de piñón manso.

Para la variable índice de durabilidad y expansión volumétrica, se verificó que briquetas confeccionadas con $100 \%$ de cáscara de piñón manso presentaron mayor durabilidad (99\%) y la vez aumentaron la expansión volumétrica en aproximadamente $734 \%$ en relación a briquetas fabricadas con $50 \%$ bagazo de caña de azúcar $+50 \%$ cáscara de piñón manso.

\section{DISCUSIÓN}

Características fisicas y energéticas de la biomasa. Los resultados constatados sobre la densidad de la biomasa de la cáscara de piñón manso y el bagazo de caña de azúcar, probablemente se deben a la cantidad de espacio intersticial existente en la cáscara de piñón manso, debido a que es un compuesto mucho más rígido que el bagazo de caña de azúcar, con sus átomos y estructuras más cercanos entre sí, que en media posee $20 \%$ de fibra, además de hemicelulosa, celulosa, lignina y otros compuestos orgánicos (Dias et al. 2009). Entretanto, el bagazo por ser un material or-
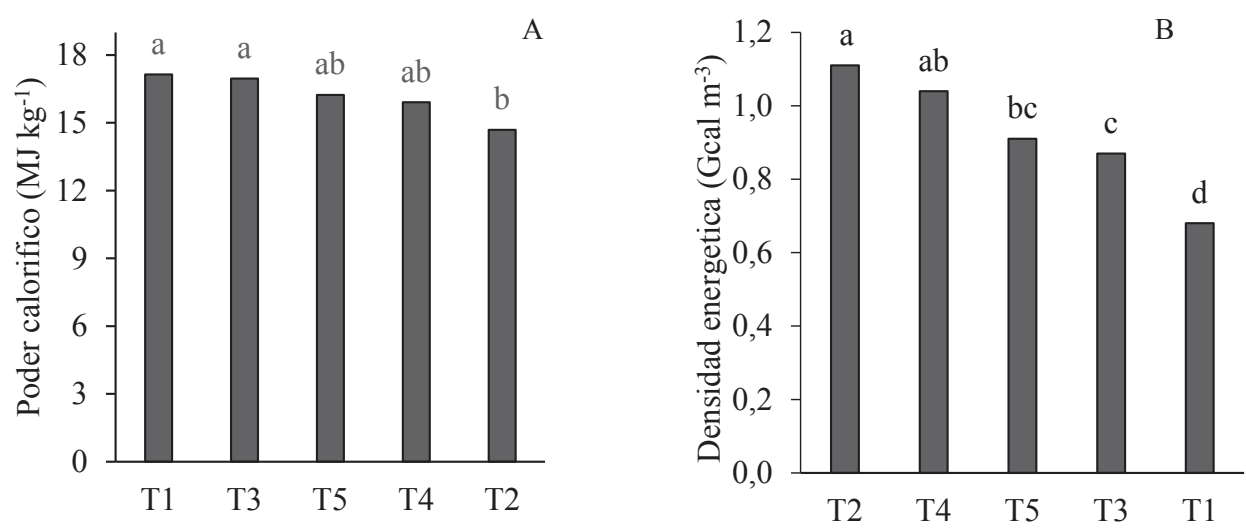

Figura 1. Valores medios de poder calorífico $\left(\mathrm{MJ} \mathrm{kg}^{-1}\right)$ y densidad energética $\left(\mathrm{Gcal} \mathrm{m} \mathrm{m}^{-3}\right)$ de bagazo de Sacharum officinarum mezclado con residuos de cáscara de Jatropha curcas en diferentes proporciones. Medias seguidas por la misma letra minúscula, no difieren entre si al $5 \%$ de significancia por la prueba de Tukey.

Average high heating value $\left(\mathrm{MJ} \mathrm{kg}^{-1}\right)$ and energy density $\left(\mathrm{Gcal} \mathrm{m}^{-3}\right)$ of the sugar cane bagasse mixed with Jatropha curcas shell in varying proportions. The averages followed by the same letter do not differ to $5 \%$ of significance by Tukey's test.

Cuadro 1. Medias de densidad aparente (DA), densidad energética (DE), resistencia a la tracción por comprensión diametral (RTCD), durabilidad (DUR) y expansión volumétrica (EXP) de briquetas confeccionadas con diferentes proporciones de biomasa de cáscara de Jatropha curcas y bagazo de Saccharum officinarum.

Bulk density averages (DA), energy density (DE), tensile strength by diametric compression (RTCD), durability (DUR) and volumetric expansion of the produced briquettes in varying proportions of the Jatropha curcas biomass and Sacharum officinarum bagasse.

\begin{tabular}{lccccc}
\hline \multirow{2}{*}{ Tratamientos } & $\mathrm{DA}$ & $\mathrm{DE}$ & $\mathrm{RTCD}$ & $\mathrm{DUR}$ & EXP \\
\cline { 2 - 6 } & $\left(\mathrm{g} \mathrm{cm}^{-3}\right)$ & $\left(\mathrm{Gcal} \mathrm{cm}^{-3}\right)$ & $(\mathrm{Mpa})$ & $\%$ & $\%$ \\
\hline $100 \%$ bagazo de caña de azúcar (T1) & $\mathrm{NF}^{*}$ & $\mathrm{NF}$ & $\mathrm{NF}$ & $\mathrm{NF}$ & $\mathrm{NF}$ \\
$100 \%$ cáscara de piñón manso (T2) & $1,28^{\mathrm{a}}$ & $4,54^{\mathrm{a}}$ & $1,45^{\mathrm{a}}$ & $99,1^{\mathrm{a}}$ & $16,6^{\mathrm{a}}$ \\
$75 \%$ bagazo + 25\% cáscara de piñón manso (T3) & $1,19^{\mathrm{b}}$ & $4,49^{\mathrm{a}}$ & $0,91^{\mathrm{b}}$ & $99,0^{\mathrm{b}}$ & $10,1^{\mathrm{b}}$ \\
$75 \%$ cáscara piñón manso + 25\% bagazo (T4) & $1,16^{\mathrm{b}}$ & $4,48^{\mathrm{a}}$ & $0,82^{\mathrm{c}}$ & $98,7^{\mathrm{c}}$ & $6,80^{\mathrm{c}}$ \\
$50 \%$ bagazo + 50 \% cáscara de piñón manso (T5) & $1,09^{\mathrm{c}}$ & $4,43^{\mathrm{a}}$ & $0,42^{\mathrm{d}}$ & $98,6^{\mathrm{d}}$ & $1,99^{\mathrm{d}}$ \\
$\mathrm{CV}$ & 6,6 & 4,1 & 6,3 & 0,3 & 6,3 \\
Média geral & 1,2 & 4,5 & 0,90 & 98,9 & 8,9 \\
\hline
\end{tabular}

"NF- no formó la briqueta. En cada columna, letras minúsculas diferentes indican diferencias significativas (Tukey, $P<0,05$ ). 

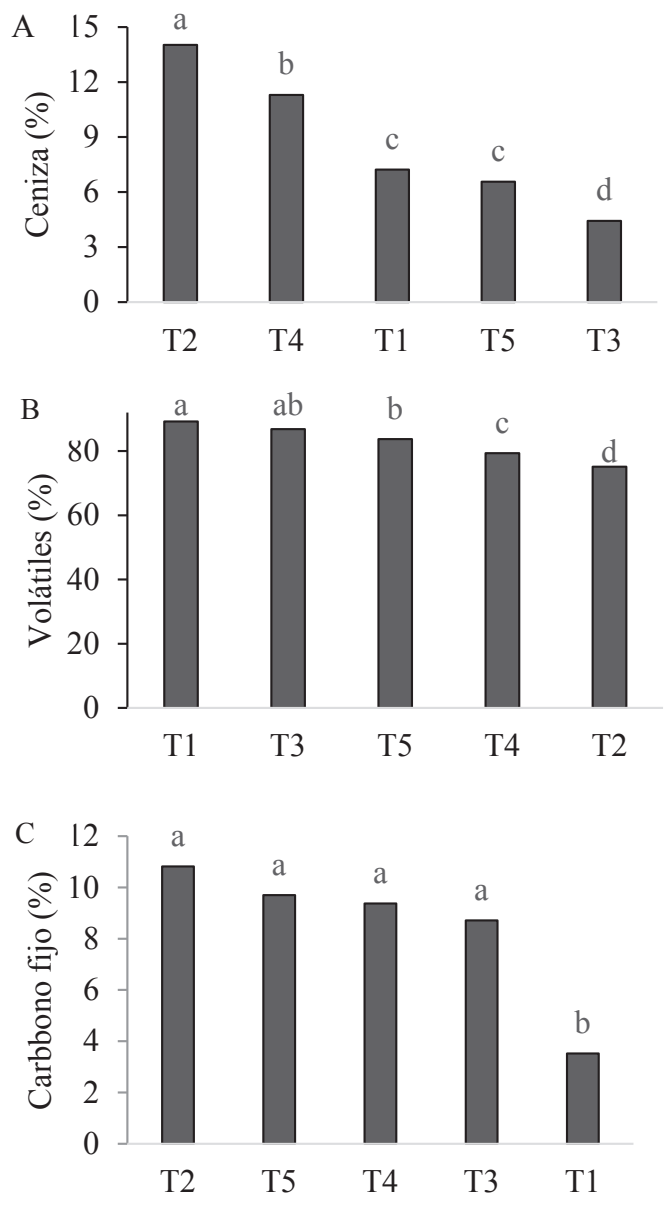

Figura 2. Valores medios de: A) contenido de cenizas (\%), B) materiales volátiles (\%) y C) carbono fijo (\%) de bagazo de $S a$ charum officinarum mezclado con residuos de Jatropha curcas en diferentes proporciones. Medias seguidas de la misma letra minúscula, no difieren entre si al $5 \%$ de significancia por la prueba de Tukey.

Average values of: A) ash content (\%), B) volatile materials $(\%)$ and C) fixed carbon content (\%) of the Sacharum officinarum bagasse mixed with Jatropha curcas shell in varying proportions. The averages followed by the same letter do not differ to $5 \%$ of significance by Tukey's test.

gánico con aproximadamente $48 \%$ de fibra permite mayor cantidad de aire en sus poros, provocando que la densidad sea menor en relación a la cáscara de piñón manso (Cortez et al. 2013).

La materia prima de baja densidad a granel, tienen efecto negativo sobre la densidad energética de la briqueta y también sobre el coste de transporte y almacenamiento, ya que la cantidad de energía por unidad de masa, es menor y la biomasa compactada ocupa mayor espacio, aumentando el costo de transporte (Forero et al. 2014). La densidad a granel en todos los tratamientos varía entre 170 a $320 \mathrm{~kg} \mathrm{~m}^{-3}$ siendo considerado un valor inferior por las normas internacionales que califican como combustibles de alta calidad, la biomasa con densidad superior a $600 \mathrm{~kg}$ $\mathrm{m}^{-3}$ (Braga 2015).

Se verifica que el bagazo de caña de azúcar (figura 1A), presenta mayor poder calorífico $\left(17,14 \mathrm{MJ} \mathrm{kg}^{-1}\right)$ en relación a la cáscara de piñón manso $\left(14,69 \mathrm{MJ} \mathrm{kg}^{-1}\right)$. Los resultados observados son inferiores a los exigidos por gran parte de las normas internacionales, entre las cuales se destaca la norma DIN-51731/PLUS de Alemania y la norma ONORM M7135 de Austria, que exige valores mayores a $18 \mathrm{MJ} \mathrm{kg}^{-1}$.

En el caso hipotético, que las briquetas fueran producidas con fines de exportación, las mismas no podrían ser exportadas para Alemania o Austria, ya que la norma internacional exigen valores de poder calorífico entre 17,5 a $19,5 \mathrm{MJ} \mathrm{kg}^{-1}$, siendo únicamente aceptadas para comercialización las briquetas confeccionadas del tratamiento 1 y 3 .

A medida se aumenta el contenido de bagazo, la densidad energética disminuye (figura 1), siendo este uno de los principales problemas del bagazo para uso energético (Cortez et al. 2013), aunque presentan mayor potencial calorífico en relación al resto de tratamientos.

En relación al contenido de cenizas (figura 2A) a medida se disminuye el contenido de bagazo, aumenta el contenido de ceniza. Esto significa, que el riesgo que la biomasa proveniente de cáscara de piñón manso al ser utilizada en forma individual, ocasione problemas de obstrucción en los equipos de combustión, es mucho más alta, si se compara con biomasa proveniente del bagazo de la caña de azúcar. La alta producción de ceniza de cáscara de piñón manso, en caso que se utilice de forma individual, podría inviabilizar un emprendimiento empresarial, debido a que debe mantenerse de forma constante, la manutención de los equipamientos, aumentando con esto los costos de producción (Paula et al. 2009). Importante destacar que el alto porcentaje de ceniza, es común en residuos agroforestales (Tomeleri et al. 2017) y no es, una característica exclusiva de la cáscara del piñón manso. El alto contenido de ceniza de la cáscara de piñón manso, en la relación al bagazo de la caña de azúcar, apenas indica una característica del material, que puede ser mejorada con la adición de otros residuos agroforestales al momento de fabricar briquetas.

En relación al contenido de materiales volátiles (figura 2B), los valores encontrados son coherentes con el rango recomendado por Ragland et al. (1991) que indica que dependiendo de la especie el material volátil pueden variar entre 70 y $90 \%$.

Importante destacar que alto contenido de material volátil favorece la combustión a menores temperaturas, pues permite que el material sea encendido más rápido, aunque provoca mayor cantidad de humo (González Martínez 2015). La combustión del tratamiento T2, T4, y T5 requiere mayor cantidades de energía y no es igual de rápida la formación de llama, en comparación con la combustión del bagazo de caña de azúcar (T1).

En relación al carbón fijo, se verifica que T2 presenta mayor porcentaje (figura $2 \mathrm{C}$ ), aunque los valores de car- 
bón fijo observados, son inferiores al reportado por Stelte et al. (2013) para pellets de madera (20-25\%). Esto indica que la biomasa compactada al momento de la combustión pierde energía, pues tanto el contenido de material volátil como el contenido de carbono fijo, son las dos formas en que se encuentra guardada la energía química en la biomasa (González Martínez 2015).

Evaluación energética y físico-mecánica de las briquetas. $\mathrm{El}$ aumento y disminución en la densidad aparente, a medida se incrementa la proporción de la cáscara de piñón manso y se reduce el contenido de bagazo de caña de azúcar (cuadro 1), se debe probablemente a que las briquetas confeccionadas con mayor proporción de bagazo, muestran mayor recuperación elástica, debido a la densidad de la partícula, que permite mayor cantidad de aire en los poros, provocando que la longitud de la briqueta aumente, luego que se retira la carga de presión de la máquina que compacta el material (Cortez et al. 2013).

Esto significa que briquetas con altos porcentajes de bagazo son inviables desde el punto de vista de transporte, almacenamiento y manoseo. Importante destacar que briquetas confeccionadas con $100 \%$ de bagazo de caña de azúcar presentaron rajaduras profundas, causadas por la compresión del pistón de la máquina compactadora, a una temperatura de compactación de $80 \pm 2{ }^{\circ} \mathrm{C}$ y presión de $140 \mathrm{kgf} \mathrm{cm}^{-2}$, lo que inviabiliza la evaluación de los parámetros físicos y químicos. Técnicamente mayor densidad aparente es importante, una vez que existe una relación directa entre el aumento de la densidad aparente y la densidad energética de la briqueta, además que proporciona mayor resistencia mecánica, volviéndose briquetas más integras al transporte o posibles caídas (Brand et al. 2014).

En relación a la densidad energética, si bien los tratamientos no difieren estadísticamente entre sí a la prueba de Tukey (cuadro 1), se observa que briquetas confeccionadas con $100 \%$ de cáscara de piñón manso son $17 \%$ más densas en relación a briquetas producidas con $50 \%$ de bagazo de caña de azúcar $+50 \%$ de cáscara de piñón manso. Esto se debe probablemente, a los compuestos químicos presentes en la biomasa de la cáscara de piñón manso que influencian drásticamente la densidad energética de la briqueta formada, principalmente porque alteran el poder calorífico de la biomasa (Dias et al. 2012).

En el caso del índice de durabilidad y la expansión volumétrica, briquetas confeccionadas con cáscara de piñón manso (cuadro 1), presentan mayor durabilidad y mayor expansión volumétrica. La literatura sugiere como adecuado una briqueta con expansión volumétrica entre 15-20\%, ya que valores superiores reducen, la temperatura del horno y la temperatura de los gases de escape por el contenido de agua presente en el material (Gonçalves et al. 2009). Además se considera que valores de humedad arriba de $15 \%$ pueden quebrar la briqueta o provocar la degradación biológica durante el transporte y almacenamiento (Dias et al. 2012).
Los resultados verificados para las diferentes características físicas y químicas de las briquetas, no desclasifican los residuos de bagazo de caña de azúcar para la producción de energía, apenas indican la necesidad de más estudios relacionados a la forma de producción, bien sea con aglomerantes o con diferentes granulometrías para densificar el material.

\section{CONCLUSIONES}

Briquetas producidas a partir de la mezcla de biomasa de caña de azúcar y cáscara de piñón manso, presentan propiedades energéticas que atienden las especificaciones de mercado, además son resistentes al transporte y almacenamiento.

Con excepción del contenido de cenizas, briquetas producidas con $100 \%$ de cáscara de piñón manso presentan índices de calidad superior a las producidas con bagazo de caña de azúcar.

\section{REFERENCIAS}

ABNT (Associação Brasileira de Normas Técnicas, BR). 1994. Argamassa e concreto: determinação da resistência à tração por compressão diametral de corpos-de-prova cilíndricos: método de ensaio. 3 p. (Norma NBR7222).

ASTM (American Society for Testing and Materials, US). 2007. Standard Test Method for Ash in Wood. 2 p. (Norma ASTM D1102-84).

ASTM (American Society for Testing and Materials, US). 2000. Standard Test Method for Gross Calorific Value of Coal and Coke by the Adiabatic Bomb Calorimeter. West Conshohocken. 8 p. (Norma D2015-00).

Beltrão EM. 2006. Considerações gerais sobre o pinhão manso ( $\mathrm{J} a-$ tropha curcas L.) e a necessidade urgente de pesquisas, desenvolvimento e inovações tecnológicas para esta planta nas condições Brasileiras. Campina Grande, Brasil. Embrapa. 4 p.

Braga M. 2015. Normas e especificações para análise de briquetes e péletes. Empresa Brasileira de Pesquisa Agricola. Consultado 15 jul. 2017. Disponible en http:// www.diadecampo.com.br/zpublisher/materias/Materia. asp? $\mathrm{id}=28322 \&$ secao $=$ Artigos $\% 20$ Especiais

Brand MA, TSF Stähelin, JC Ferreira, MD Neves. 2014. Produção de biomassa para geração de energia em povoamentos de pinus taeda 1. com diferentes idades. Revista Árvore 38(2): 353-360.

Cabral de Sousa Dias JM, D Tatiane de Souza, M Braga, M Onoyama, CH Behling Miranda, PF Dias Barbosa, JD Rocha. 2012. Produção de briquetes e péletes a partir de resíduos agrícolas, agroindustriais e florestais. Embrapa Agroenergia 1: 132 .

Cordeiro CG, RD Toledo Filho, LM Tavares, EMR Fairbairn. 2008. Pozzolanic activity and filler effect of sugar cane bagasse ash in Portland cement and lime mortars. Cement \& Concrete Composites 30(2): 410-418.

Cortez L, P Magalhaes, J Happi. 1992. Principais subprodutos da agroindústria canavieira e sua valorização. Revista Brasileira de Energia 2(2): 1-17.

Dias Júnior AF, AM de Andrade, SS Costa Júnior . 2014. Ca- 
racterização de briquetes produzidos com resíduos agroflorestais. Pesquisa Florestal Brasileira 34(79): 225-228. doi: 10.4336/2014.pfb.34.79.613

Dias Vieira de Souza A, S Palma Fávaro, LC Vinhas Ítavo, R Roscoe. 2009. Caracterização química de sementes e tortas de pinhão manso, nabo forrageiro e crambe. Pesquisa Agropecuaria Brasileira 44(10): 1328-1335.

DIN (Deutsches Institut Fur Normung, GE). 1996. Testing of solid fuels-Compressed untreated wood, requirements and testing. 3 p. (Norma DIN 51731).

Dias A, D Souza, M Braga, M Onoyama, C Miranda, D Barbosa, D Rocha. 2012. Produção de briquetes e péletes a partir de resíduos agrícolas, agroindustriais e florestais. Embrapa Agroenergia 1: 132.

Forero-Nuñez CA, G Camargo-Vargas, FE Sierra-Vargas. 2014. Modelos de compresión aplicados al proceso de densificación de combustibles sólidos binarios carbón-madera. Itecknne 11(1): 196-203.

Gonçalves JE, MP Sartori, AL Leão. 2009. Energia de briquetes produzidos com rejeitos de resíduos sólidos urbanos e madeira de Eucalyptus grandis. Revista Brasileira de Engenharia Agrícola e Ambiental 13(5): 657-661.

González Martínez ID. 2013. Potencial del aserrín generado en la transformación primaria de Pinus Patula para la fabricación de Pellets. Tesis de Maestría en Ciencias Forestales. Chapingo, México. Facultad de Ciencias Forestales, Universidad Autónoma de Chapingo. 116 p.

Lucena DA, R Medeiros, UT Fonseca, PS Assis. 2008. Aglomeração de moinha de carvão vegetal e sua possível aplicação em alto-forno e geração de energia. Tecnologia em Metalúrgica e Materiais 4(4): 1-6.

Oliveira E, M Faria, A Evangelista, P Melo. 2012. Resposta do pinhão-manso à aplicação de níveis de irrigação e doses de adubação potássica. Revista Brasileira de Engenharia Agrícola e Ambiental 16(6): 539-598.

Paula M, I Tinôco, C Rodrigues, E Silva, C Souza. 2009. Potential of sugarcane bagasse ash as a partial replacement material for Portland cement. Revista Brasileira de Engenharia Agrícola e Ambiental 13(3): 353-357.

Protásio TP, IC Alves, PF Trugilho, VO Silva, A Baliza. 2011. Compactação de biomassa vegetal visando à produção de biocombustíveis sólidos. Pesquisa Florestal Brasileira 31(68): 273-283.

Ragland KW, DJ Aerts, AJ Baker. 1991. Properties of wood for combustion analysis. Bioresource technology 37(2): 161168.

Soares L, V Moris, F Yamaji, M Paiva. 2015. Utilização de resíduos de borra de café e serragem na moldagem de briquetes e avaliação de propriedades. Revista Materia 20(2): 550560.

Stelte W, K Nielsen, HO Hansen, J Dahl, L Shang, AR Sanadi. 2013. Reprint of pelletizing properties of torrefied wheat straw. Biomass and Bioenergy 53(1): 105-112.

Tomeleri J, LB Valentim, JP Silva, FM Yamaji, FA Pádua. 2017. Caracterização Química e Energética de Epicarpo Residual do Pinhão Manso (Jatropha curcas L.) e Briquete Produzido. Revista Virtual de Quimica 9(3): 942-952.

Vale A, M Mendes, M Amorim, F Dantas F. 2011. Potencial energético da biomassa e carvão vegetal do epicarpo e da torta de pinhão manso (Jatropha curcas). Revista Cerne 17(2): 267-273.

Yamaji FM, L Vendrasco, W Chrisostomo, WP Flores. 2013. Análise do comportamento higroscópico de briquetes. Revista Energía na Agricultura 28(1): 11-15. 
